DOI: 10.12957/demetra.2016.21777

\title{
Pistas implícitas e obesidade: estratégias de proteção contra o marketing de alimentos
}

\section{Implicit cues and obesity: protection strategies against food marketing}

Isabel Antunes David'

Laura Krutman'

Jéssica Rocha de Andrade?

Renata Lopes Araújo $0^{3}$

Filipe Braga ${ }^{2,4}$

Fábio da Silva Gomes ${ }^{5,6}$

Mirtes Garcia Pereira?

Letícia de Oliveira'

Sonia Gleiser ${ }^{2}$

M. Carmen Fernández-Santaella ${ }^{7}$

Rafael Delgado ${ }^{7}$

Eliane Volchan ${ }^{2,3}$

\footnotetext{
'Universidade Federal Fluminense, Instituto Biomédico. Rio de Janeiro-RJ, Brasil.

${ }^{2}$ Universidade Federal do Rio de Janeiro, Instituto de Psiquiatria. Rio de Janeiro-RJ, Brasil.

${ }^{3}$ Universidade Federal do Rio de Janeiro, Instituto de Biofísica Carlos Chagas Filho. Rio de Janeiro-RJ, Brasil.

${ }^{4}$ Universidade Federal do Rio de Janeiro, Campus Macaé. Macaé-RJ, Brasil.

${ }^{5}$ Unidade Técnica de Alimentação, Nutrição e Câncer. Instituto Nacional do Câncer José Alencar Gomes da Silva (INCA), Ministério da Saúde. Rio de Janeiro-RJ, Brasil. ${ }^{6}$ New York University, Department of Nutrition, Food Studies, and Public Health. NY, USA.

${ }^{7}$ Universidad de Granada, Facultad de Psicología. GR, Espanha.
}

Financiamento: Programa Jovem Cientista do Nosso Estado. Faperi. Processo n ${ }^{0}$ E-26/201.506/2014. Programa Cientista do Nosso Estado. Faperi. Processo $n^{0}$ E-26/102.294/2013. Programa de Cooperação Internacional Capes/ DGU. Capes. Processo n ${ }^{0}$ 290/13.

\section{Correspondência / Correspondence}

Isabel A. David

Universidade Federal Fluminense. Instituto Biomédico/ MFL Rua Hernani Pires de Melo, 101, São Domingos, NiteróiRJ, Brasil. CEP 24210-130.

E-mail: isabeldavid@id.uff.br

\section{Resumo}

A proteção contra o marketing de alimentos não saudáveis constitui importante estratégia de controle da obesidade e de doenças crônico-degenerativas. A indústria de alimentos aplica técnicas de neuromarketing como forma de persuasão implícita e promoção dos seus produtos, assim torna-se urgente a aplicação deste conhecimento no âmbito das estratégias de defesa, aumentando suas chances de sucesso. Propõe-se, com base em diversos estudos já realizados, agregar o conhecimento científico interdisciplinar na fundamentação teórica das ações públicas voltadas para a promoção da alimentação saudável.

Palavras-chave: Obesidade. Marketing. Publicidade de Alimentos. Mecanismos de Defesa. Propaganda. Psicologia. Neurobiologia. Saúde Pública.

\section{Abstract}

Protection against marketing of unhealthy food is an important strategy to curb obesity and chronic non-communicable diseases. Given that food industry applies neuromarketing techniques as a form of implicit promotion, the use of such knowledge to ground defense strategies becomes urgent and increases their chances of success. We propose, based on several studies, an interdisciplinary research effort to guide public actions to promote healthy eating.

Key words: Obesity. Marketing. Food Publicity. Defense Mechanisms. Advertisement. Psychology. Neurobiology. Public Health. 


\section{Introdução}

A obesidade é considerada um grave problema de saúde pública mundial, sendo apontada como importante fator para a mortalidade e morbidade de doenças cardiovasculares, diabetes, câncer e outras doenças crônico-degenerativas. ${ }^{1}$ Tal tendência de aumento da obesidade é particularmente importante em indivíduos jovens. Nos últimos trinta anos, a porcentagem de crianças e adolescentes com sobrepeso ou sob risco de se tornar obeso aumentou substancialmente em todo o mundo. ${ }^{2} \mathrm{O}$ Brasil vem atravessando uma transição nutricional que se reflete em mudanças relacionadas aos hábitos alimentares da população. As alterações observadas estão bem caracterizadas pela menor ocorrência de desnutrição e pela maior incidência de obesidade na população, a qual vem crescendo de forma consistente, sobretudo na população de mais baixa renda. ${ }^{3}$ Inquéritos populacionais realizados no período entre 1974-1975 e 2008-2009 demonstraram que as prevalências de excesso de peso e de obesidade aumentam continuamente em ambos os sexos. ${ }^{4}$

Este quadro mundial de tendência à obesidade parece estar relacionado tanto à falta de atividade física como ao padrão alimentar. ${ }^{5}$ Como agravo, observa-se grande oferta de gêneros alimentícios com elevadas quantidades de açúcar, gorduras e sódio pela indústria alimentícia. Autoridades em saúde acreditam que o acúmulo de mensagens embutidas nas propagandas desses alimentos pode induzir o maior consumo destes. ${ }^{6-8}$

Nos Estados Unidos, crianças entre doise 11 anos veem diariamente aproximadamente 12 propagandas de TV relacionadas a produtos alimentícios ricos em gordura, açúcar e/ou sódio. ${ }^{9}$ No Brasil, as crianças são expostas a duas propagandas de alimentos não saudáveis a cada hora, contendo em sua maioria produtos com alto valor calórico e baixo valor nutricional. ${ }^{10,11}$ Outro trabalho aponta para a presença de elementos discutíveis quanto à questão ética neste tipo de propaganda no Brasil. ${ }^{12}$

Diversas soluções têm sido propostas para atenuar a influência do marketing de alimentos não saudáveis, que vão desde a proibição de toda a publicidade voltada para crianças ${ }^{13}$ até mecanismos mais brandos e inefetivos como a autorregulação. ${ }^{14}$ Vale ressaltar que a Declaração Política da Reunião de Alto Nível da Assembleia Geral da ONU sobre a prevenção e controle de doenças crônicas não transmissíveis, aprovada em janeiro de 2012, aponta que estas doenças lideram as causas evitáveis de mortalidade e morbidade em todo o mundo. Segundo esta Declaração, a prevenção é o foco principal, e entre as principais estratégias preventivas está a regulação da publicidade de alimentos e bebidas dirigida às crianças. ${ }^{15}$

Neste artigo, revisaremos o papel das pistas ambientais sobre o comportamento de consumo de alimentos, visando melhor compreender o impacto do marketing de alimentos sobre o comportamento de crianças, adolescentes e adultos. Sequencialmente, discutiremos como esses 
conceitos podem servir de subsídios para estratégias de Saúde Coletiva que visem proteger as pessoas dessa indesejada influência.

\section{Pistas ambientais e o comportamento de consumo}

Os fatores motivacionais que iniciam, sustentam e direcionam nosso comportamento podem ser implicitamente guiados por pistas do ambiente. Por exemplo, Bargh \& Chartrand, ${ }^{16}$ em uma série de estudos empíricos, demonstraram que a exposição prévia a determinado estímulo pode influenciar a resposta do indivíduo a um estímulo subsequente, sem que exista consciência do indivíduo sobre tal influência. Parte das respostas comportamentais aos estímulos que nos cercam seria automática, sendo necessário um grande esforço cognitivo para tomar decisões conscientes em um ambiente favorável a pistas implícitas. ${ }^{16,17}$

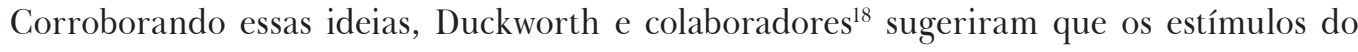
ambiente seriam contínua e inintencionalmente avaliados desde sua percepção como positivos ou negativos, afetando implicitamente as respostas afetivas, cognitivas e comportamentais. Em termos biológicos, pesquisas em psicofisiologia da emoção demonstram que a relevância motivacional de um estímulo emocional pode ativar circuitos motores e preparar o indivíduo para a ação de forma automática. ${ }^{19-21}$

Trabalhos no âmbito do consumo alimentar têm demonstrado que pistas ambientais influenciam significativamente o comportamento alimentar. ${ }^{22} \mathrm{O}$ tamanho e a cor da embalagem, o tamanho do prato e da porção, o contexto, a variedade e a quantidade de alimento disponíveis são apenas alguns exemplos de fatores ambientais que podem influenciar o consumo alimentar de forma implícita. ${ }^{23,24}$

O trabalho de Roefs e colaboradores,${ }^{25}$ por exemplo, demonstrou uma associação implícita entre o contexto e a escolha de alimentos através de uma tarefa comportamental de tempo de reação. Os voluntários eram mais rápidos em responder palavras positivas precedidas de palavras de alimentos ricos em gordura em um contexto envolvendo pistas sobre a palatabilidade dos alimentos. Em contrapartida, em um contexto envolvendo pistas de saúde, os tempos de resposta para as palavras positivas eram menores quando as palavras precedentes eram de alimentos saudáveis.

Outro trabalho, utilizando a técnica de eletroencefalografia, demostrou associação positiva entre a atividade cortical beta frontal e o relato de palatabilidade a alimentos doces quando estes eram consumidos em um contexto de cafeteria, indicando uma facilitação motora nesse contexto. ${ }^{26}$ O comportamento de outras pessoas é outra pista importante, uma vez que os indivíduos imitam implicitamente o comportamento de outros indivíduos, incluindo a escolha de um alimento ou a quantidade consumida, sem a percepção de que estão fazendo isto..$^{27,28}$ 
A indústria alimentícia utiliza pistas ambientais que associam seus produtos a aspectos positivos e sedutores, predispondo ações voltadas à aproximação desses alimentos. Muitas das práticas de marketing de alimentos voltadas para crianças, adolescentes e adultos vão muito além da persuasão explícita para convencê-los de que produtos de uma marca são melhores do que de outra. Para capturar os consumidores, incorporam técnicas de neuromarketing, tendo como embasamento teórico a Psicologia e a Neurobiologia, para sutilmente influenciar as preferências alimentares e de consumo, por exemplo, através do estabelecimento de associações de marca com emoções positivas.

De fato, a elaboração de comerciais sofisticados, com imagens positivas, além do uso de displays em estabelecimentos, com ótima iluminação, localização e fácil acesso, gera impulso de compra e consumo para adultos e, principalmente, para as crianças e adolescentes. Já foi demonstrado que a propaganda predispõe o consumo automático de produtos alimentícios, impossibilitando qualquer controle por parte do consumidor atingido por esta propaganda. ${ }^{29} \mathrm{~A}$ literatura, portanto, sugere que a exposição à publicidadede alimentos possui efeitos diretos potencialmente poderosos.

Recentes estudos experimentais demonstram que a exposição à publicidade de alimentos na televisão aumenta as calorias consumidas durante e imediatamente após a exposição. ${ }^{30-32}$ Nesses estudos, os participantes não tinham conhecimento de que foram afetados pela publicidade. Pistas contidas na propaganda publicitária (por exemplo, a imagem de comer, preferências de gosto, ou identificação com os atores ou personagens do produto) poderiam aumentar o consumo através da ativação de ações automáticas. ${ }^{33}$

A exposição a outras formas de marketing, menos complexas (por exemplo, logotipos, marcas ou banners na internet), também pode criar efeitos similares. ${ }^{29}$ Todos os indivíduos expostos sofrem influência dessa manipulação, mas crianças e adolescentes são particularmente hipervulneráveis. ${ }^{30}$ Agravando o problema, alimentos insalubres, contendo altas quantidades de sódio, açúcar, gordura saturada e trans, proporcionam alta durabilidade, palatabilidade e baixa saciedade, sendo os principais produtos comercializados e presentes nas propagandas..$^{10,11}$

Há um número crescente de estudos que demonstram os efeitos causais diretos da exposição à publicidade de alimentos na dieta das crianças e jovens, como: o aumento no consumo de lanches e no total de calorias; menor consumo de frutas e hortaliças; e maiores taxas de obesidade. ${ }^{32,34,35}$ No trabalho de Harris, Bargh \& Brownell, ${ }^{31}$ crianças em idade escolar foram expostas a programas de TV, contendo ou não propaganda de alimentos com alto teor calórico e baixo valor nutricional, enquanto seguravam um pacote de biscoito do tipo snacks. As crianças expostas às propagandas consumiram $45 \%$ mais biscoitos durante o programa do que as não expostas. Outro estudo mostrou que o consumo de alimentos insalubres aumenta em crianças expostas à propaganda de alimentos, ${ }^{36}$ inclusive aquelas entre cinco e sete anos de idade. ${ }^{37}$ De forma preocupante, as crianças obesas parecem ser mais afetadas pela exposição à propaganda de alimentos, aumentando de forma substancial e significativa o consumo de snacks com alto conteúdo calórico, gordura saturada e/ou açúcar. ${ }^{38}$ 
O gasto do brasileiro com alimentação corresponde a aproximadamente $20 \%$ do seu orçamento. ${ }^{39} \mathrm{O}$ investimento da indústria de alimentos em publicidade, sobretudo voltada para o público jovem, reflete a importância deste mercado: três bilhões de reais somente no primeiro semestre de 2014. ${ }^{40}$ A maior parte das propagandas promove produtos que os jovens só deveriam consumir em quantidades muito limitadas ou não deveriam consumir. ${ }^{11}$ Estas práticas de marketing agressivo, voltado principalmente para crianças e adolescentes, atua, portanto, de forma perversa, infligindo o direito dos jovens e da população em geral a uma alimentação adequada e propícia à saúde nutricional.

\section{Estratégias de proteção contra o marketing de alimentos não saudáveis}

Considerando-se o acúmulo de evidências, a influência negativa da publicidade de alimentos sobre a saúde da população torna-se óbvia. De fato, a Declaração Política da Assembleia das Nações Unidas, citada anteriormente, e a Resolução da 63ํㅡㄹ Assembleia da Organização Mundial da Saúde (OMS) convergem para o reconhecimento da extensa evidência de que os produtos com altos teores de sal, açúcar e gorduras saturadas e trans são os principais fatores de risco para obesidade e doenças crônicas não transmissíveis, e propõem ação global para redução deste impacto pernicioso. ${ }^{41}$

Neste sentido, o debate público e científico deve ir além da questão de saber se o marketing de alimentos afeta negativamente a saúde, para um debate sobre como nos proteger de sua influência óbvia. O modelo de defesa ao marketing de alimentos proposto por Harris e colaboradores ${ }^{6}$ tem como base os conhecimentos em Psicologia e propõe quatro condições necessárias para que os indivíduos sejam capazes de resistir aos estímulos do marketing de alimentos: 1) a consciência, incluindo a atenção consciente aos estímulos de marketing individuais e compreensão da sua intenção persuasiva; 2) a compreensão dos efeitos resultantes da exposição a estímulos e como se defender eficazmente contra esses efeitos; 3) a capacidade, incluindo a capacidade cognitiva e os recursos disponíveis para resistir eficazmente; e 4) a motivação, ou o desejo de resistir.

De acordo com esse modelo, a capacidade de resistir à influência da publicidade de alimentos depende das diferentes formas de marketing, assim como dos contextos em que estas ocorrem. O modelo considera que recursos cognitivos adicionais são necessários para inibir o impulso direcionado a produtos alimentares extremamente tentadores, mas não saudáveis, comumente apresentados na publicidade de alimentos. Além disso, ele reconhece que as crianças e jovens são particularmente difíceis de proteger, sobretudo pela pouca motivação em resistir à influência do marketing, uma vez que a capacidade de resistir vai se desenvolvendo com a idade e compreensão. ${ }^{30}$

Dentro da perspectiva de como pistas ambientais influenciam o comportamento alimentar, sugere-se que a exposição repetida à publicidade de alimentos pode alterar diretamente crenças e 
comportamentos, sem o processamento ativo deliberado das informações apresentadas. Portanto, seria difícil combater os efeitos implícitos da exposição à comercialização de alimentos. ${ }^{6}$

Propostas de defesa da população contra esse tipo de persuasão são desafiantes e urgentes. O uso, pela indústria de alimentos, de métodos experimentais controlados no âmbito da Psicologia e Neurobiologia para identificar práticas eficazes de comercialização de alimentos não saudáveis suscita especial preocupação entre os defensores da Saúde Pública. Como a indústria de alimentos utiliza cada vez mais os métodos e as teorias científicas para analisar o que funciona, ${ }^{42}$ a necessidade de investigação dentro da mesma esfera para examinar como se defender contra tais práticas se torna ainda mais crítica.

Algumas soluções para a crise de obesidade infantil têm sido propostas, como as medidas de autorregulação ${ }^{14}$ e de educação alimentar. ${ }^{43}$ As medidas de autorregulação publicitária não oferecem proteção suficiente. Já é bastante evidente a inefetividade de medidas voluntárias, ${ }^{44} \mathrm{o}$ que tem respaldado a recomendação por medidas mandatórias. Medidas de proteção mais efetivas devem ser aplicadas, a fim de evitar a interferência nos direitos de outras pessoas à alimentação e à saúde. A seguir, serão discutidas em mais detalhe algumas medidas propostas e/ou aplicadas.

\section{Campanhas informativas}

As campanhas de mídia de massa são consideradas pela OMS umas das mais importantes estratégias de prevenção e controle de doenças crônico-degenerativas. ${ }^{45}$ As campanhas de conscientização da população abrangem desde o marketing e campanhas sociais para promover os alimentos saudáveis, até a formulação de guias alimentares para a população. ${ }^{46}$

Alguns estudos têm demonstrado que estratégias criativas de educação nutricional parecem surtir algum efeito. Por exemplo, pré-escolares consomem mais alimentos saudáveis quando estes recebem nomes especiais. ${ }^{47}$ No entanto, propagandas apenas informativas e pouco criativas parecem surtir pouco efeito. A campanha promovida pelo governo da Austrália, utilizando vários vetores da mídia para promover hábitos de vida mais saudáveis, parece ter aumentado o conhecimento da população sobre a relação entre circunferência abdominal e doenças crônicas. No entanto, não se observoua mudança dos hábitos da população, como o aumento do consumo de alimentos saudáveis e atividade física. ${ }^{48}$

Outra estratégia informativa inclui a implementação de guias alimentares. Estes visam apresentar para a população, de forma mais clara e resumida, quais seriam as principais recomendações nutricionais promotoras da saúde. Existe, no entanto, a dificuldade em formular e padronizar guias de fácil compreensão e com o potencial de atingir as necessidades nutricionais da maior parte da população. ${ }^{43}$ Além disso, são poucas as evidências quanto à eficiência dos guias alimentares atualmente propostos. ${ }^{49}$ 
Outro fato a ser considerado é que os governos não conseguem investir tanto tempo e recursos na promoção de dietas saudáveis quanto a indústria de alimentos investe na divulgação comercial de produtos nocivos à saúde. De forma geral, também faltam evidências de que o marketing voltado para os alimentos saudáveis possa se refletir na redução do consumo dos alimentos altamente palatáveis, como os ultraprocessados. Determinar se o marketing de alimentos saudáveis pode ser usado para compensar os efeitos da comercialização de alimentos pouco saudáveis parece ser, portanto, outra pergunta crítica a ser investigada.

\section{Os movimentos contrapropaganda}

Práticas comuns da indústria de alimentos exploram o fato de os processos sociais ainda estarem em desenvolvimento nos jovens, tornando-os mais passíveis à ameaça de exclusão social. Existem evidências de que as pistas de isolamento social são consideradas extremamente alarmantes e dolorosas, ${ }^{50,51}$ e que a sensação de solidão inclusive prevê o aumento da morbidade e da mortalidade. ${ }^{52,53}$ Imagens de interação social são capazes de pré-ativar circuitos motores que propiciam movimentos de interação social como o carinho, ${ }^{20}$ ressaltando a importância deste tipo de pista. Imagens de famílias e bebês felizes, de sucesso social e de celebridades são maciçamente empregadas em propaganda de alimentos não saudáveis. Resistir a estas pistas, portanto, torna-se uma tarefa difícil, mesmo estando consciente dessas influências.

A conscientização, por parte da população, dos efeitos persuasivos e nocivos do marketing de alimentos pode fornecer uma importante estratégia, gerando movimentos sociais que se contrapõem ao efeito nocivo do marketing de alimentos não saudáveis. Os movimentos sociais têm um papel não só informativo, mas inclusivo, uma vez que reduzem a sensação de isolamento social daqueles que se opõem ao conteúdo das propagandas, o que pode aumentar a motivação para resistir.

São exemplo disto os movimentos de contrapropaganda como os proporcionados pela Fundação Legado Americano, denominado "a verdade" ("The Truth ${ }^{\circledast}$ "), que ganharam destaque nas campanhas antitabagistas. ${ }^{54}$ A característica principal deste movimento, criado em 1999, era expor a verdade por trás das campanhas publicitárias promovidas pela indústria tabagista, aumentando a consciência e a compreensão sobre a intenção persuasiva embutida na propaganda de produtos do tabaco. O mais interessante é o fato de este movimento antitabagista estar nucleado em uma população jovem, uma vez que o público adolescente é o alvo da indústria tabagista, como exposto em documentos internos da indústria. ${ }^{55}$ Há evidências mostrando que os movimentos sociais de contrapropaganda voltados aos produtos do tabaco são capazes de promover mudanças na atitude e comportamento de jovens e adultos, contribuindo para a redução do consumo desses produtos. ${ }^{54}$ 
No âmbito dos produtos alimentícios não saudáveis, este tipo de movimento ainda é tímido. Diferentemente dos produtos do tabaco, que são inegavelmente prejudiciais à saúde, estes produtos abrem margem para a dúvida sobre que aspectos nutricionais poderiam ser prejudiciais à saúde. A indústria de alimentos mascara o conteúdo insalubre de seus produtos (como excesso de gordura saturada, presença de gordura trans, excesso de açúcar, presença de aditivos cancerígenos e excesso de sódio) através, por exemplo, da adição de itens supostamente tidos como saudáveis, como fibras, vitaminas e minerais. Estes últimos apresentam-se em destaque nas embalagens (e.g. "zero gordura trans", "sem colesterol”, "zero açúcar", "mais fibras”), contrapondo-se à tabela de valores nutricionais, que é de difícil compreensão para o público em geral. Além disso, a indústria alimentícia busca apoio em profissionais de saúde, sociedades da área médica e em atletas, visando dar credibilidade a algum aspecto saudável do seu produto. Estudos mostram que adultos julgam como mais saudáveis produtos que utilizam estas abordagens, em relação a outros na realidade mais saudáveis, porém sem tais destaques. ${ }^{56}$

Dentro desse contexto, expor a verdade à população quanto ao conteúdo nutricional real de cada produto e aos possíveis malefícios à saúde torna-se urgente e essencial. Dixon e colaboradores ${ }^{57}$ testaram o efeito da contrapropaganda sobre a percepção de pais quanto ao conteúdo não saudável de produtos alimentícios. A estratégia utilizada envolvia a apresentação da embalagem do produto como este é comercializado, enfatizando algum aspecto saudável (e.g. integral). A mesma embalagem era então apresentada, enfatizando-se agora o aspecto não saudável (e.g. 30\% açúcar). Eles mostraram que a contrapropaganda foi eficiente em reduzir a intenção de compra e a confiabilidade na propaganda. Outro estudo mostrou que a contrapropaganda de alimentos não saudáveis em forma de banner animado foi capaz de afetar pré-adolescentes, aumentando a percepção de insalubridade do produto e reduzindo a confiabilidade na propaganda. ${ }^{58}$

Os achados no âmbito da contrapropaganda voltados para os produtos alimentícios não saudáveis ainda são escassos, além de faltarem estudos com abordagens neurobiológicas. No entanto, a aplicação da contrapropaganda parece ter o potencial de permitir uma melhor análise crítica das mensagens embutidas nas propagandas de alimentos por crianças, adolescentes e adultos, além de se contrapor às estratégias de ameaça de exclusão social promovida pelas propagandas.

\section{0 uso de advertências sanitárias}

A introdução, no Brasil, de advertências nas embalagens dos cigarros contribuiu para uma considerável redução do número de fumantes no país. Os resultados positivos são traduzidos na diminuição da proporção de fumantes na população. ${ }^{59}$ No caso da campanha antitabagista focada na utilização de imagens pictóricas nas embalagens de produtos do cigarro, o respaldo científico se fez de grande valia. Neste sentido, uma equipe multidisciplinar formada por pesquisadores da 
Universidade Federal Fluminense e da Universidade Federal do Rio de Janeiro, em parceria com INCA, ANVISA e o Departamento de Artes \& Design da PUC-Rio, forneceram embasamento teórico e experimental no campo da psicofisiologia da emoção para a seleção de categorias de imagens que seriam mais adequadas para as advertências e para os testes da sua eficácia. ${ }^{60-62}$ Recentemente, demostrou-se que as advertências pictóricas utilizadas atualmente no Brasil foram capazes de gerar uma predisposição implícita para o afastamento do cigarro em fumantes, opondose ao apelo positivo promovido pela embalagem do cigarro. ${ }^{61}$

Apesar da crescente evidência em favor do uso de advertências aplicadas a produtos do tabaco, inclusive no âmbito da Psicologia e Neurobiologia, pouco tem sido feito no sentido de aplicar estratégias semelhantes no combate à propaganda de alimentos não saudáveis.

No Reino Unido, foi proposto um semáforo nutricional, que utiliza diferentes cores como indicativo de valores elevados ou seguros de açúcar, sódio, gordura saturada e trans. Segundo Temple \& Fraser ${ }^{63}$ esse é o melhor modelo de rotulagem até o momento, pois apresenta os nutrientes-chave de forma simples e inteligível. Os autores também revelam que a maioria dos consumidores tem dificuldade em compreender a informação fornecida pelos rótulos nutricionais tradicionais e traduzila em orientações práticas para a seleção de uma dieta saudável. Portanto, o semáforo nutricional aumenta consideravelmente a capacidade dos consumidores de avaliar a salubridade dos alimentos.

Estudos recentes têm mostrado que a rotulagem em forma de semáforo disposta nas embalagens dos produtos alimentícios é capaz de afetar as escolhas feitas pelo consumidor no momento da compra $\cdot{ }^{64-67} \mathrm{~A}$ implementação de um sistema de rotulagem em forma de semáforo é capaz de aumentar as vendas de itens rotulados como saudáveis (verde) e reduzir as vendas de itens insalubres (vermelho) ${ }^{67}$ incluindo indivíduos de diferentes origens raciais / étnicas e socioeconômicas. ${ }^{65}$ Além disso, a rotulagem em forma de semáforo parece aumentar a porcentagem de consumidores que apontam a saúde e a nutrição como importante fator de escolha de alimentos. ${ }^{66} \mathrm{Em}$ outro trabalho, demonstrou-se que os participantes se dispunham a pagar mais por produtos saudáveis que continham rótulos em forma de semáforos nutricionais em comparação a rótulos puramente informativos. ${ }^{68}$

Além das evidências comportamentais, trabalhos com medidas neurobiológicas também têm explorado a eficiência do semáforo nutricional. Enax e colaboradores ${ }^{68}$ compararam dois tipos de rótulos nutricionais: um puramente baseado em informações e outro em forma de semáforo. Nesse estudo, encontrou-se que semáforos nutricionais vermelhos ativaram o córtex pré-frontal dorsolateral, que é uma região implicada na inibição de resposta e no autocontrole durante a escolha de alimentos. Por sua vez, o semáforo nutricional verde ativou o córtex cingulado posterior, que está possivelmente envolvido no processamento de expectativa de recompensa. E outro estudo, utilizando a técnica de monitoramento ocular, mostrou que a incorporação de cores aumentou a saliência e reduziu o tempo de deteç̧ão dos rótulos nutricionais. ${ }^{69}$ 
O Brasil também tem investido na regulação de produtos não saudáveis, mais concretamente a partir da resolução RDC no 24, promulgada em 15 de junho de 2010, pela Agência Nacional de Vigilância Sanitária (ANVISA), para regulamentar a promoção comercial de alimentos com quantidades elevadas de açúcar, de gordura saturada, de gordura trans, de sódio e de bebidas com baixo teor nutricional. ${ }^{70}$ A resolução institui que a divulgação e a promoção comercial desses alimentos e bebidas sejam acompanhadas de advertências em forma de frases, contendo informações sobre o excesso desses componentes e sobre os riscos à saúde.

Estudos anteriores demonstraram que textos antecedendo estímulos visuais emocionais modulam as respostas eletrofisiológicas, a reatividade cardíaca, a sudorese e a resposta hemodinâmica a estas imagens. ${ }^{71-73}$ Mais especificamente no âmbito alimentar, Grabenhorst, Rolls \& Bilderbeck ${ }^{74}$ observaram, através da técnica de ressonância magnética funcional, que ativações relacionadas ao valor afetivo do sabor umami na região orbitofrontal (área implicada no processamento de recompensa) podem ser realçadas por textos descritivos apresentados aos participantes. Outro estudo mostrou que participantes expostos a rótulos, que ressaltavam tanto o sabor como os aspectos saudáveis de uma maçã, a selecionaram significativamente mais comparados aos participantes que viram a maçã sem qualquer descritor. ${ }^{75}$ Portanto, algumas evidências no âmbito da Neurobiologia apontam para a modulação das respostas autonômicas e dos circuitos cerebrais por frases textuais.

As advertências e rotulagens informativas têm o potencial de se contrapor ao impacto de imagens positivas embutidas nas propagandas ou embalagens de alimentos não saudáveis. No entanto, são necessários mais estudos que embasem a elaboração e implementação de tais estratégias ${ }^{76}$ É importante ainda considerar a interdisciplinaridade desses estudos. As pesquisas no âmbito da Psicologia e Neurobiologia devem ser previamente planejadas para responder perguntas que visem atender às necessidades práticas e imediatas no âmbito da saúde pública, reduzindo tempo e recursos.

\section{Conclusão}

A compreensão detalhada dos processos psicológicos e neurobiológicos implicados nas estratégias de marketing pode ajudar os setores de saúde a determinar os métodos mais eficazes de proteger a população contra essa influência indesejada. É importante desenvolver habilidades para melhorar a defesa contra as influências nocivas do marketing de alimentos não saudáveis, alertando os pais, educadores e autoridades de saúde para as formas de comercialização, e como elas afetam crianças e adolescentes.

Além disso, é preciso ainda limitar as práticas de marketing e agregar o conhecimento científico interdisciplinar para desenvolver ações de promoção da alimentação saudável e inibição do consumo de alimentos não saudáveis. 


\section{Referências}

1. Lim SS, Vos T, Flaxman AD, Danaei G, Shibuya K, Adair-Rohani H, et al. A comparative risk assessment of burden of disease and injury attributable to 67 risk factors and risk factor clusters in 21 regions, 19902010: a systematic analysis for the Global Burden of Disease Study 2010. Lancet 2012; 380(9859):2224-60.

2. Ng M, Fleming T, Robinson M, Thomson B, Graetz N, Margono C, et al. Global, regional, and national prevalence of overweight and obesity in children and adults during 1980-2013: a systematic analysis for the Global Burden of Disease Study 2013. Lancet 2014; 6736(14):1-16.

3. Monteiro CA, Conde WL, Popkin BM. Income-specific trends in obesity in Brazil: 1975-2003. Am J Public Health 2007; 97(10):1808-12.

4. Instituto Brasileiro de Geografia e Estatística. Pesquisa de orçamentos familiares: 2008-2009. Antropometria e Estado Nutricional de crianças, adolescentes e adultos. Brasília: IBGE; 2010.

5. Mokdad AH, Marks JS, Stroup DF, Gerberding JL. Actual causes of death in the United States, 2000. JAMA 2004; 291(10):1238-45.

6. Harris JL, Brownell KD, Bargh JA. The food marketing defense model: integrating psychological research to protect youth and inform public policy. Soc Issues Policy Rev. 2009; 3(1):211-71.

7. Powell LM, Szczypka G, Chaloupka FJ. Adolescent exposure to food advertising on television. Am. J. Prev. Med. 2007; 33(4 Suppl.):251-6.

8. Fiates GMR, Amboni RDMC, Teixeira E. Television use and food choices of children: Qualitative approach. Appetite 2008; 50(1):12-8.

9. Powell LM, Schermbeck RM, Szczypka G, Chaloupka FJ, Braunschweig CL. Trends in the nutritional content of TV food advertisements seen by children in the US: analyses by age, food categories and companies. Arch. Pediatr. Adolesc Med. 2011; 165(12):1078-86.

10. Almeida SDS, Nascimento PCB, Quaioti TCB. Quantidade e qualidade de produtos alimentícios anunciados na televisão brasileira. Rev. Saude Publica 2002; 36(3):353-5.

11. Kelly B, Halford JCG, Boyland EJ, Chapman K, Bautista-Castaño I, Berg C, et al. Television food advertising to children: a global perspective. Am. J. Public Health 2010; 100(9):1730-6.

12. Henriques P, Sally EO, Burlandy L, Beiler RM. Regulation of publicity for children's food as a strategy for promotion of health. Cien. Saude Colet. 2012; 17(2):481-90.

13. Brasil. Conselho Nacional dos Direitos da Crianças e do Adolescente. Resolução no 163, de 13 de março de 2014. Dispõe sobre a abusividade do direcionamento de publicidade e de comunicação mercadológica à criança e ao adolescente. [acesso em: 15 out. 2015]. Diário Oficia da União 04 abr. 2014; Seção 1(65):4. Disponível em: http://pesquisa.in.gov.br/imprensa/jsp/visualiza/index.jsp?jor nal $=1 \&$ pagina $=4 \&$ data $=04 / 04 / 2014$

14. Sharma LL, Teret SP, Brownell KD. The food industry and self-regulation: Standards to promote success and to avoid public health failures. Am J Public Health 2010; 100(2):240-6.

15. United Nations. Political Declaration of the high-level meeting of the general assembly on the prevention and control of non-communicable diseases. 201213 p. [acesso em: 6 out. 2015]. Disponível em: http://www.who.int/nmh/events/un_ncd_summit2011/political_declaration_en.pdf 
16. Bargh JA, Chartrand TL. The unbearable automaticity of being. Am. Psychol. 1999; 54(7):462-79.

17. Ferguson M, Hassin R, Bargh J. Implicit motivation: past, present, and future. In: James Y. Shah WLG, editor. Handbook of motivation science. NY: Guilford; 2008. p. 150-66.

18. Duckworth KL, Bargh JA, Garcia M, Chaiken S. The automatic evaluation of novel stimuli. Psychol Sci. 2002; 13(6):513-9.

19. Pereira MG, Oliveira L, Erthal FS, Joffily M, Mocaiber IF, Volchan E, et al. Emotion affects action: midcingulate cortex as a pivotal node of interaction between negative emotion and motor signals. Cogn Affect Behav. Neurosci. 2010; 10(1):94-106.

20. Campagnoli RR, Krutman L, Vargas CD, Lobo I, Oliveira JM, Oliveira L, et al. Preparing to caress: a neural signature of social bonding. Front Psychol. 2015; 6(16):1-9.

21. Oliveira LAS, Imbiriba LA, Russo MM, Nogueira-Campos AA, Rodrigues EC, Pereira MG, et al. Preparing to grasp emotionally laden stimuli. PLoS One 2012; 7(9):e45235.

22. Cohen D, Farley TA. Eating as an automatic behavior. Prev. Chronic Dis. 2008; 5(1):A23.

23. Wansink B. Environmental factors that increase the food intake and consumption volume of unknowing consumers. Annu. Rev. Nutr. 2004; 24(217):455-79.

24. Wansink B, Painter JE, Lee Y-K. The office candy dish: proximity's influence on estimated and actual consumption. Int. J. Obes. (Lond). 2006; 30(5):871-5.

25. Roefs A, Quaedackers L, Werrij MQ, Wolters G, Havermans R, Nederkoorn C, et al. The environment influences whether high-fat foods are associated with palatable or with unhealthy. Behav Res Ther. 2006; 44(5):715-36.

26. Yoshimura H, Honjo M, Sugai T, Kawabe M, Kaneyama K, Segami N, et al. Influences of audiovisual environments on feelings of deliciousness during having sweet foods: an electroencephalogram frequency analysis study. Nutr. Neurosci. 2011; 14(5):210-5.

27. Hermans RCJ, Lichtwarck-Aschoff A, Bevelander KE, Herman CP, Larsen JK, Engels RCME. Mimicry of food intake: The dynamic interplay between eating companions. PLoS One 2012; 7(2):1-6.

28. Hermans RCJ, Larsen JK, Lochbuehler K, Nederkoorn C, Herman CP, Engels RCME. The power of social influence over food intake: examining the effects of attentional bias and impulsivity. Br. J. Nutr. 2013; 109 (3):572-80.

29. Fitzsimons GM, Chartrand TL, Fitzsimons GJ. Automatic effects of brand exposure on motivated behavior: how apple makes you think different. J. Consum. Res. 2008; 35(1):21-35.

30. Harris JL, Pomeranz JL, Lobstein T, Brownell KD. A crisis in the marketplace: how food marketing contributes to childhood obesity and what can be done. Annu. Rev. Public Health 2009; 30:211-25.

31. Harris JL, Bargh JA, Brownell KD. Priming effect of television food advertising on eating behavior. Heal Psychol. 2010; 28(4):404-13.

32. Boyland EJ, Halford JCG. Television advertising and branding. Effects on eating behaviour and food preferences in children. Appetite 2013; 62:236-41. 
33. Bargh JA, Morsella E. Unconscious Behavioral Guidance Systems. In: Agnew CR, Carlston DE, Graziano WG, Kelly JR, editores. Then a miracle occurs: focusing on behavior in social psychological theory and research. New York: Oxford University Press; 2009. p. 89-118.

34. Boyland EJ, Harrold J a, Kirkham TC, Corker C, Cuddy J, Evans D, et al. Food commercials increase preference for energy-dense foods, particularly in children who watch more television. Pediatrics 2011; 128(1):e93-100.

35. Barr-Anderson DJ, Larson NI, Nelson MC, Neumark-Sztainer D, Story M. Does television viewing predict dietary intake five years later in high school students and young adults? Int. J. Behav. Nutr. Phys. Act. 2009; 6:1-8.

36. Halford JC, Gillespie J, Brown V, Pontin EE, Dovey TM. Effect of television advertisements for foods on food consumption in children. Appetite 2004; 42(2):221-5.

37. Halford JCG, Boyland EJ, Hughes G, Oliveira LP, Dovey TM. Beyond-brand effect of television (TV) food advertisements/commercials on caloric intake and food choice of 5-7-year-old children. Appetite 2007; 49(1):263-7.

38. Halford JC, Boyland EJ, Hughes GM, Stacey L, McKean S, Dovey TM. Beyond-brand effect of television food advertisements on food choice in children: the effects of weight status. Public Health Nutr. 2008; 11(9):897-904.

39. Instituto Brasileiro de Geografia e Estatística. Pesquisa de orçamentos familiares 2008-2009: análise da disponibilidade domiciliar de alimentos e do estado nutricional no Brasil. Brasília: IBGE; 2010. 54 p. 0

40. IBOPE. Almanaque IBOPE. Setores econômicos 2014. [acesso em: 01 jul. 2016]. Disponível em: $<$ https://www.kantaribopemedia.com/setores-economicos-2014/>.

41. World Health Organization. Set of recommendations on the marketing of foods and nonalcoholic beverages to children. Geneva: WHO; 2010. Disponível em: http://apps.who.int/iris/ bitstream/10665/44416/1/9789241500210_eng.pdf

42. Ait Hammou K, Galib MH, Melloul J. The contributions of neuromarketing in marketing research. J. Manag. Res. 2013; 5(4):20.

43. Hawkes C. Promoting healthy diets through nutrition education and changes in the food environment: an international review of actions and their effectiveness. Rome: Nutrition Education and Consumer Awareness Group, Food and Agriculture Organization of the United Nations; 2013.

44. Hebden LA, King L, Grunseit A, Kelly B, Chapman K. Advertising of fast food to children on Australian television: the impact of industry self-regulation. Med J Aust. 2011; 195(1):20-4.

45. World Health Organization. Global status report on non-communicable Diseases 2010. Geneva: WHO; 2011. 163 p.

46. Brasil. Ministério da Saúde. Secretaria de Atenção à Saúde. Departamento de Atenção Básica. Guia alimentar para a população brasileira. 2a ed. Brasília: Ministério da Saúde; 2014.

47. Wansink B, Just DR, Payne CR, Klinger MZ. Attractive names sustain increased vegetable intake in schools. Prev Med (Baltim). 2012; 55(4):330-2. 
48. King EL, Grunseit AC, O’Hara BJ, Bauman AE. Evaluating the effectiveness of an Australian obesity mass-media campaign: How did the "Measure-Up" campaign measure up in New South Wales? Health Educ. Res. 2013; 28(6):1029-39.

49. Keller I, Lang T. Food-based dietary guidelines and implementation: lessons from four countries Chile, Germany, New Zealand and South Africa. Public Health Nutr. 2008; 11(08):867-74.

50. Eisenberger NI, Lieberman MD, Williams KD. Does rejection hurt? An FMRI study of social exclusion. Science. 2003; 302(5643):290-2.

51. Eisenberger NI. Broken Hearts and Broken Bones: a neural perspective on the similarities between social and physical pain. Curr. Dir. Psychol. Sci. [Internet]. 2012; 21(1):42-7. Disponível em: https:// sanlab.psych.ucla.edu/wp-content/uploads/sites/31/2015/05/Eisenberger2012CDPS.pdf

52. Hare TA, Malmaud J, Rangel A. Focusing attention on the health aspects of foods changes value signals in vmPFC and improves dietary choice. J. Neurosci. 2011; 31(30):11077-87.

53. Cacioppo JT, Patrick W. Loneliness: human nature and the need for social connection. New York: W. W. Norton \& Company; 2009.

54. Allen JA, Vallone D. The truth campaign: using countermarketing to reduce youth smoking. In: Healey BJ, R. Zimmerman Jr RS, editores. The new world of health promotion: new program development, implementation, and evaluation. Sudbury, MA: Jones \& Bartle; 2009. p. 195-216.

55. World Health Organization. The tobacco industry documents: what they are, what they tell us, and how to search them. a practical manual. [Internet]. [acesso em: 10 jul. 2015]. Disponível em: http:// wwwlive.who.int/entity/tobacco/publications/industry/TI_manual_content.pdf

56. Dixon H, Scully M, Wakefield M, Kelly B, Chapman K, Donovan R. Parent's responses to nutrient claims and sports celebrity endorsements on energy-dense and nutrient-poor foods: an experimental study. Public Health Nutr. 2011;14(6):1071-9.

57. Dixon H, Scully M, Kelly B, Donovan R, Chapman K, Wakefield M. Counter-advertising may reduce parent's susceptibility to front-of-package promotions on unhealthy foods. J. Nutr. Educ. Behav. 2014; 46(6):467-74.

58. Dixon H, Scully M, Kelly B, Chapman K, Wakefield M. Can counter-advertising reduce pre-adolescent children's susceptibility to front-of-package promotions on unhealthy foods?: Experimental research. Soc. Sci. Med. 2014; 116:211-9.

59. Brasil. Ministério da Saúde. Secretaria de Vigilancia em Saúde. Vigitel Brasil 2011: Vigilância de fatores de risco e proteção para doenças crônicas por inquérito telefônico. Brasília: Ministério da Saúde; 2012. 131 p.

60. Nascimento BEM, Oliveira L, Vieira A, Joffily M, Gleiser S, Pereira MG, et al. Avoidance of smoking: the impact of warning labels in Brazil. Tob Control 2008; 17(6):405-9.

61. Volchan E, David IA, Tavares G, Nascimento BM, Oliveira JM, Gleiser S, et al. Implicit motivational impact of pictorial health warning on cigarette packs. PLoS One 2013; 8(8):4-9. 
62. Nascimento BEM, Gamba Jr N, Oliveira O, Pereira MG, Spitz R, Gleiser S, et al. Neurosciences, graphic arts, and public health: new health warnings on cigarette packaging. História, Ciências, Saúde-Manguinhos 2010; 17:243-52.

63. Temple NJ, Fraser J. Food labels: a critical assessment. Nutrition 2014; 30(3):257-60.

64. Lacanilao RD, Cash SB, Adamowicz WL. Heterogeneous Consumer responses to snack food taxes and warning labels. J. Consum. Aff. 2011; 45(1):108-22.

65. Levy DE, Riis J, Sonnenberg LM, Susan J, Thorndike AN. Food choices of minority and low-income employees. Am. J. Prev. Med. 2013; 43(3):240-8.

66. Sonnenberg L, Gelsomin E, Levy DE, Riis J, Barraclough S, Thorndike AN. A traffic light food labeling intervention increases consumer awareness of health and healthy choices at the point-ofpurchase. Prev. Med. (Baltim) 2013; 57(4):253-7.

67. Thorndike AN, Sonnenberg L, Riis J, Barraclough S, Levy DE. A 2-phase labeling and choice architecture intervention to improve healthy food and beverage choices. Am. J. Public Health 2012; 102(3):527-33.

68. Enax L, Hu Y, Trautner P, Weber B. Nutrition labels influence value computation of food products in the ventromedial prefrontal cortex. Obesity 2015; 23(4):786-92.

69. Antúnez L, Giménez A, Maiche A, Ares G. Influence of interpretation Aids on attentional capture, visual processing, and understanding of front-of-package nutrition labels. J. Nutr. Educ. Behav. 2015; 47(4):292-9.e1.

70. Brasil. Agência Nacional de Vigilância Sanitária. Resolução ANVISA no 24/2010, que dispõe sobre a oferta, propaganda, publicidade, informação e outras práticas correlatas cujo objetivo seja a divulgação e a promoção comercial de alimentos considerados com quantidades elevadas de açúcar, de gordura saturada, de gordura trans, de sódio e de bebidas com baixo teor nutricional. Diário Oficial da União 29 jun. 2010; Seção 1(122): 46-47. [acesso em: 1 out. 2015]. Disponível em: http://portal.anvisa.gov. br/documents/33880/2568070/RDC_24_2010.pdf/21beffdc-0188-4f81-a710-32935fa5f67b

71. Mocaiber I, Pereira MG, Erthal FS, Machado-Pinheiro W, David IA, Cagy M, et al. Fact or fiction? An event-related potential study of implicit emotion regulation. Neurosci. Lett. 2010; 476(2):84-8.

72. Mocaiber I, Sanchez TA, Pereira MG, Erthal FS, Joffily M, Araujo DB, et al. Antecedent descriptions change brain reactivity to emotional stimuli: a functional magnetic resonance imaging study of an extrinsic and incidental reappraisal strategy. Neuroscience 2011; 193:241-8.

73. Oliveira LAS, Oliveira L, Joffily M, Pereira-Junior PP, Lang PJ, Pereira MG, et al. Autonomic reactions to mutilation pictures: Positive affect facilitates safety signal processing. Psychophysiology 2009; 46(4):870-3.

74. Grabenhorst F, Rolls ET, Bilderbeck A. How cognition modulates affective responses to taste and flavor: top-down influences on the orbitofrontal and pregenual cingulate cortices. Cereb Cortex 2008; 18(7):1549-59. 
75. Forwood SE, Walker AD, Hollands GJ, Marteau TM. Choosing between an apple and a chocolate bar: the impact of health and taste labels. PLoS One 2013; 8(10):1-5.

76. Hieke S, Taylor CR. A critical review of the literature on nutritional labeling. J. Consum Aff. 2012; 46(1):120-56.

Recebido: $26 / 2 / 2016$

Revisado: 04/4/2015

Aceito: 28/5/2016 